\title{
Agregação, carbono e nitrogênio em agregados do solo sob plantio direto com integração lavoura-pecuária
}

\author{
Arcângelo Loss ${ }^{(1)}$, Marcos Gervasio Pereira(1), Simone Guimarães Giácomo ${ }^{(1)}$, \\ Adriano Perin ${ }^{(2)}$ e Lúcia Helena Cunha dos Anjos ${ }^{(1)}$
}

(1)Universidade Federal Rural do Rio de Janeiro, Departamento de Solos, BR 465, Km 7, CEP $23890-000$ Seropédica, RJ. E-mail: arcangeloloss@yahoo.com.br, gervasio@ufrrj.br, sigiacomo@yahoo.com.br, lanjosrural@gmail.com (2)Instituto Federal de Educação, Ciência e Tecnologia Goiano, Campus Rio Verde, Rodovia Sul Goiana, Km 01, Zona Rural, CEP $75901-970$ Rio Verde, GO. E-mail: perinrj@yahoo.com.br

Resumo - O objetivo deste trabalho foi avaliar a estabilidade dos agregados, o conteúdo de matéria orgânica leve do solo (MOL), a distribuição dos teores de carbono orgânico total (COT) e nitrogênio (N) e a abundância natural de ${ }^{13} \mathrm{C}$ e ${ }^{15} \mathrm{~N}$ dos agregados de um Latossolo Vermelho argiloso sob sistema plantio direto (SPD) com integração lavoura-pecuária (ILP), em comparação à uma área em SPD sem ILP e uma área de Cerrado natural em Montividiu, GO. Foram coletadas amostras de solo nas camadas de $0-5$ e 5-10 cm, em delineamento inteiramente casualizado. Foram avaliados distribuição da massa dos agregados, diâmetro médio ponderado (DMP), diâmetro médio geométrico (DMG) e MOL, e foram quantificados os teores de COT, $\mathrm{N}, \delta^{13} \mathrm{C}$ e $\delta^{15} \mathrm{~N}$ dos agregados. A área de Cerrado apresentou os maiores valores de DMP, DMG e MOL. O SPD-ILP apresentou valores maiores de DMP, DMG, COT, N e MOL na camada 5-10 cm do que o SPD, sem braquiária. O SPD-ILP promoveu aumentos nos índices de agregação do solo (0-5 e 5-10 cm), nos teores de MOL $(5-10 \mathrm{~cm})$, COT e N $(0-5 \mathrm{~cm})$ e na formação de agregados estáveis em água $(5-10 \mathrm{~cm})$, em comparação ao SPD, sem braquiária.

Termos para indexação: carbono orgânico total, Cerrado, índices de agregação, matéria orgânica leve, milho + braquiária, rotação de culturas.

\section{Aggregation, carbon and nitrogen in soil aggregates under no-tillage with crop-livestock integration}

\begin{abstract}
The objective of this work was to evaluate the stability of aggregates, light organic matter content (LOM), distribution of total organic carbon (TOC) and nitrogen $(\mathrm{N})$ contents, and natural abundance of ${ }^{13} \mathrm{C}$ and ${ }^{15} \mathrm{~N}$ in aggregates of a clayed Oxisol under no-tillage (NT) with crop-livestock integration (CLI), in comparison to an area under NT without CLI, and an area of natural Cerrado, in Montividiu, GO, Brazil. Soil samples were collected at the layers of $0-5$ and $5-10 \mathrm{~cm}$, in a completely randomized block design. The mass distribution of aggregates, mean weight diameter (MWD), geometric mean diameter (GMD), and LOM were evaluated, and the levels of TOC, $\mathrm{N}, \delta^{13} \mathrm{C}$ and $\delta^{15} \mathrm{~N}$ aggregates were quantified. The Cerrado area had the highest values of MWD, GMD and LOM. The NT-CLI showed higher values of MWD, GMD, TOC, N and LOM in the layer of $5-10 \mathrm{~cm}$ than the area of NT without brachiaria. The NT-CLI increased the indices of soil aggregation ( $0-5$ and $5-10 \mathrm{~cm})$, the levels of LOM $(5-10 \mathrm{~cm})$, TOC and N $(0-5 \mathrm{~cm})$ and the formation of water-stable aggregates $(5-10 \mathrm{~cm})$ in comparison to the NT without palisade grass.
\end{abstract}

Index terms: total organic carbon, Cerrado, aggregation index, light organic matter, corn + palisadegrass, crop rotation.

\section{Introdução}

O monocultivo e práticas culturais inadequadas têm causado perda de produtividade e degradação do solo e dos recursos naturais. A reversão desse quadro pode ser conseguida por meio de tecnologias, como o sistema de plantio direto (SPD), com preparo mínimo do solo e prática de rotação de culturas, e os sistemas de integração lavoura-pecuária (ILP) (Moreti et al., 2007; Macedo, 2009).
A utilização de pastagens em áreas degradadas de lavouras para a melhoria das propriedades edáficas do solo, pela presença de palha e raízes da pastagem, aumenta os teores de carbono e melhora significativamente as condições de aeração e capacidade de infiltração de água. Outro importante objetivo da ILP é a produção de pasto e forragens para a alimentação animal na estação seca; neste caso, há muitas variações no país, em decorrência do clima e dos solos de cada região. No conjunto, a ILP torna-se 
fundamental para a sustentabilidade e a produtividade do sistema agropecuário, o que possibilita a redução de custos pelo menor uso de insumos e diversificação, tanto da atividade agrícola quanto da pecuária, com aumento de renda e diminuição de problemas ambientais (Gonçalves \& Franchini, 2007).

Na ILP em SPD, os benefícios aportados por este sistema, tais como a manutenção da estrutura do solo e o aumento dos teores de matéria orgânica, entre outros, são potencializados pela introdução de espécies forrageiras. No geral, essas forrageiras acumulam mais carbono do que as culturas agrícolas, cuja fitomasssa é, muitas vezes, insuficiente para a manutenção da cobertura do solo (Especial Embrapa, 2009).

Várias culturas têm sido utilizadas nos sistemas de ILP, entre as quais destacam-se: soja, milho, milheto, sorgo, nabo forrageiro, girassol, algodão e gramíneas forrageiras tropicais, principalmente as braquiárias (Urochloa sp.), consorciadas ou não. Entretanto, poucos experimentos de longa duração de rotação lavoura-pecuária em ecossistemas tropicais e subtropicais têm sido relatados (Macedo, 2009; Franchini et al., 2010). Assim, são necessários mais estudos sobre esse sistema em relação às propriedades edáficas, com destaque aos teores de carbono e nitrogênio do solo e dos agregados.

A hipótese deste trabalho é que o SPD, associado à utilização da braquiária junto à rotação de culturas (ILP-SPD), pode aumentar os índices de agregação do solo e os teores de carbono e nitrogênio dos agregados, em comparação à uma área em SPD, sem braquiária.

O objetivo deste trabalho foi avaliar a estabilidade dos agregados, o conteúdo de matéria orgânica leve do solo, a distribuição dos teores de carbono orgânico total e nitrogênio e a abundância natural $d e{ }^{13} \mathrm{C}$ e ${ }^{15} \mathrm{~N}$ dos agregados de um Latossolo Vermelho sob sistema de plantio direto (SPD) com integração lavoura-pecuária (ILP), em comparação à uma área em SPD sem ILP e uma área de Cerrado natural em Montividiu, GO.

\section{Material e Métodos}

O experimento foi conduzido na Fazenda Vargem Grande, da Agropecuária Peeters, localizada em Montividiu, GO $\left(17^{\circ} 21^{\prime} \mathrm{S}, 51^{\circ} 28^{\prime} \mathrm{W}\right)$. O clima da região apresenta duas estações bem definidas: uma seca (maio a setembro) e outra chuvosa (outubro a abril). A temperatura média anual varia entre $20 \mathrm{e}$ $28^{\circ} \mathrm{C}$. O solo da área foi classificado como Latossolo
Vermelho Distrófico típico textura argilosa (Santos et al., 2006), com 657, 214 e $129 \mathrm{~g} \mathrm{~kg}^{-1}$ de argila, areia e silte, respectivamente. Na composição mineralógica da fração argila, predominam gibbsita, caulinita e hematita.

Anteriormente à realização das avaliações, existia uma cobertura vegetal de Cerradão que foi derrubada em 1975, para implantação de pastagens (Urochloa decumbens (Stapf) R.D. Webster); esta atividade permaneceu na área por dez anos. Em seguida, a área foi manejada na forma de sistema convencional, com cultivo de grãos por sete anos.

Os sistemas avaliados consistiram de duas áreas cultivadas em SPD por 17 anos: uma área somente com rotação de culturas (girassol-soja-milhoalgodão) de coordenadas $17^{\circ} 21^{\prime} 7^{\prime \prime} \mathrm{S}, \quad 51^{\circ} 29^{\prime} 26^{\prime \prime} \mathrm{W}$, a $958 \mathrm{~m}$ de altitude, e outra com braquiária (U. ruziziensis) em consórcio com milho safrinha (milho-braquiária-feijão-algodão-soja), para intensificar a produção de palhada no período seco do ano, de coordenadas $17^{\circ} 21^{\prime} 51^{\prime \prime} \mathrm{S}, 51^{\circ} 28^{\prime} 36^{\prime \prime} \mathrm{W}$, a $859 \mathrm{~m}$ de altitude. Nesta área, foi utilizado o sistema ILP, com milho e braquiária plantados simultaneamente. Após a colheita do milho, foi feita a introdução de bovinos na área $\left(2,0 \mathrm{UA} \mathrm{ha}^{-1}\right)$, com permanência dos animais por 90 dias (julho a setembro). Depois da remoção do gado, permaneceram as touceiras de braquiária, e esperou-se as primeiras chuvas para realizar a adubação em cobertura na braquiária, com $200 \mathrm{~kg} \mathrm{ha}^{-1}$ da fórmula NPK (20-00-20) a lanço na primeira quinzena de setembro. Após a rebrota, quando a área apresentou-se totalmente coberta pela capineira, foi feita a dessecação e o plantio do feijão. Uma área de Cerrado (Cerradão) natural adjacente às áreas cultivadas (17²6'39"S, 5122'30"W, a $951 \mathrm{~m}$ de altitude) foi considerada como condição original do solo.

As áreas eram cultivadas com girassol em SPD (girassol-soja-milho-algodão) e milho + braquiária em SPD-ILP (milho-braquiária-feijão-algodão-soja) quando da coleta de amostras. Por ocasião do plantio, foi realizada, na cultura do milho, a aplicação de $400 \mathrm{~kg} \mathrm{ha}^{-1}$ da fórmula NPK (08-20-10) na semeadura $+200 \mathrm{~kg} \mathrm{ha}^{-1}(20-00-20)$ na cobertura a lanço quando o milho apresentou entre seis e oito folhas $+100 \mathrm{~kg} \mathrm{ha}^{-1}$ de ureia a lanço quando o milho estava com dez folhas. Para a cultura do girassol, utilizou-se $300 \mathrm{~kg} \mathrm{ha}^{-1}$ da fórmula NPK (05-20-10) + $2 \mathrm{~kg} \mathrm{ha}^{-1}$ de boro na 
semeadura $+150 \mathrm{~kg} \mathrm{ha}^{-1}(20-00-20)$ na cobertura, quando o girassol apresentou de quatro a seis folhas.

Para a coleta das amostras, em março de 2008, foi delimitada uma área em torno de $600 \mathrm{~m}^{2}$, na qual foram abertas quatro trincheiras transversais às linhas de semeadura em cada área. A densidade do solo (Ds) foi avaliada nas profundidades de $0-5$ e $5-10 \mathrm{~cm}$ com auxílio de anel volumétrico (Claessen, 1997). Nestas trincheiras, nas mesmas profundidades, também foram coletados blocos indeformados para avaliação da estabilidade dos agregados, com auxílio de espátula. As amostras foram secas ao ar e, em seguida, passadas por peneiras de 8 e $4 \mathrm{~mm}$ de diâmetro de malha, segundo Claessen (1997). Para a avaliação da distribuição dos agregados, foram pesadas $25 \mathrm{~g}$ das amostras que ficaram retidas na peneira de $4 \mathrm{~mm}$. Posteriormente, as amostras foram transferidas para um jogo de peneiras com malhas de 2,00, 1,00,0,50,0,25 e 0,105 mm, umedecidas com pulverizador e submetidas à agitação vertical no aparelho de Yooder (Yooder, 1936), durante 15 min. Após o tempo determinado, o material retido em cada peneira foi retirado, separado com o auxílio de jato d'água, colocado em placas de petri e secado em estufa a $60^{\circ} \mathrm{C}$, até massa constante.

Com os dados de massa de agregados, foram calculados diâmetro médio ponderado (DMP) e diâmetro médio geométrico (DMG) dos agregados. Por meio da massa dos agregados, avaliou-se a distribuição dos agregados por classes de diâmetro médio $\quad(8,0-2,0 ; \quad 2,0-1,0 ; \quad 1,0-0,5 ; \quad 0,5-0,25$; 0,25-0,105 mm).

Para a quantificação da matéria orgânica leve (MOL), as amostras coletadas nas trincheiras $(0-5$ e $5-10 \mathrm{~cm})$ foram peneiradas em peneira de $2,00 \mathrm{~mm}$. A MOL foi avaliada pelo método da flotação (Anderson \& Ingram, 1989).

Os teores de carbono orgânico total (COT) e N total dos agregados do solo $(0-5 \mathrm{~cm})$ foram determinados pelo método de combustão a seco, em um autoanalisador CHN-600 Carlo Erba EA-1110 de C e N, a $900^{\circ} \mathrm{C}$, (Carlo Erba Reagenti, Milan, Itália) e a abundância natural de ${ }^{13} \mathrm{C}$ e ${ }^{15} \mathrm{~N}(0-5 \mathrm{~cm})$ foi avaliada com espectrômetro de massa Delta Plus (Finnigan Mat, Bremen, Alemanha). As medidas de $\delta^{15} \mathrm{~N}$ e $\delta^{13} \mathrm{C}$ e a determinação do percentual de COT e $\mathrm{N}$ foram realizadas no Laboratório de Ecologia Isotópica do Centro de Energia Nuclear na Agricultura, Piracicaba,
SP. Com os dados de COT e N, calculou-se a relação $\mathrm{C} / \mathrm{N}$.

Utilizou-se o delineamento inteiramente casualizado. Foram avaliadas a normalidade dos dados (Lilliefors) e a homogeneidade das variâncias (Bartlett, 1936). Os resultados foram submetidos à análise de variância com teste $\mathrm{F}$, e os valores médios comparados entre si pelo teste t, a $5 \%$ de probabilidade.

\section{Resultados e Discussão}

Os maiores valores de Ds foram encontrados nas áreas em SPD (girassol-soja-milho-algodão) e SPD-ILP (milho-braquiária-feijão-algodão-soja) e o menor na área de Cerradão, independentemente da profundidade analisada (Tabela 1). O manejo realizado na área em SPD provavelmente contribuiu para a compactação do solo, padrão não observado para a área de Cerradão, que não foi submetida à ação antrópica.

Entre as áreas em SPD, a com ILP apresentou menor valor de Ds, nas duas profundidades avaliadas. Este padrão pode ser atribuído ao consórcio com braquiária, que possui um sistema radicular bem desenvolvido, o que favorece a agregação do solo e o maior aporte de matéria orgânica pelo sistema radicular. Assim, há um aumento da porosidade do solo e, consequentemente, uma redução nos valores de Ds. Estes resultados corroboram os de Fonseca et al. (2007), que, ao avaliar os atributos físicos, químicos e biológicos de Latossolo em Cerrado com duas rotações de culturas, verificaram que a área com braquiária apresentou os menores valores de Ds.

Em um estudo da evolução da qualidade física do solo após um ano de ILP em Querência, MT, Franchini et al. (2010) verificaram, por meio da determinação da resistência à penetração (RP), que o uso de forrageiras tropicais (Urochloa sp.) em sistemas de ILP melhorou a qualidade física do solo e proporcionou, em um ano, a descompactação de camadas compactadas em virtude do uso contínuo do solo com soja. A partir dos resultados obtidos por Franchini et al. (2010), podese inferir que a diminuição da RP está, possivelmente, associada à diminuição dos valores de Ds.

Quanto aos índices de estabilidade de agregados estáveis em água, DMP e DMG, verificou-se que, independentemente da profundidade, a área de Cerradão apresentou os maiores valores (Tabela 1) em decorrência do maior aporte de material vegetal na 
área, que se encontrava em condição original do solo, sem interferência de qualquer forma de cultivo. Houve aumento dos teores de MOS e, consequentemente, aumento da agregação do solo. Os maiores teores de MOL encontrados na área de Cerradão $(0-5 \mathrm{~cm})$ demonstram o maior aporte de material vegetal nesta área em comparação às áreas em SPD.

Entre as áreas cultivadas, nas duas profundidades avaliadas, a área em SPD-ILP apresentou valores maiores de DMP e DMG do que a área em SPD, sem braquiária. Portanto, o uso da braquiária (SPD-ILP) propiciou boa cobertura vegetal ao solo, o que impede ou diminui a ação direta das gotas de chuva e mantém mais uniforme a umidade e a temperatura do solo; além disso, acarreta melhor desenvolvimento do sistema radicular das culturas e maior atividade microbiana, que contribuem para a criação de um ambiente mais favorável à agregação do solo, em comparação à área em SPD, sem braquiária. Salton et al. (2008) obtiveram resultados semelhantes, ao avaliar a agregação e a estabilidade de agregados do solo em sistemas agropecuários em Latossolo, no Mato Grosso do Sul. Os autores observaram que os sistemas de manejo do solo com rotação de lavoura e pastagem (U. brizantha) em SPD favoreceram a formação de agregados estáveis de maior tamanho, em comparação à sistemas apenas com lavouras, sem braquiária.

Os maiores valores de MOL foram encontrados na área de Cerradão na profundidade de $0-5 \mathrm{~cm}$, e não foram observadas diferenças entre as áreas em SPD (Tabela 1). Na profundidade de 5-10 cm, a área em SPD-ILP apresentou valores iguais aos da área de Cerradão, e valores maiores que a área em SPD. Os valores de MOL indicam que no Cerradão há maior aporte de serapilheira em comparação às áreas cultivadas $(0-5 \mathrm{~cm})$. Entretanto, na profundidade de 5-10 cm, a ILP favoreceu o aporte de resíduos das raízes em subsuperfície e a renovação do sistema radicular; o aporte equiparou-se ao da serapilheira produzida na área de Cerradão, e foi mais eficiente que o da área em SPD (sem braquiária) em aumentar os teores de MOL no solo. Estes resultados corroboram os de Pereira et al. (2010), que encontraram maiores teores de MOL em áreas em SPD cultivadas com milho e soja sobre os resíduos vegetais da braquiária, em comparação ao cultivo destas mesmas culturas sobre os resíduos de milheto e crotalária, em solo de Cerrado, em Uberaba, MG.

Em relação à distribuição da massa dos agregados estáveis em água, observou-se que, na profundidade de 0-5 cm, a área de Cerradão apresentou maiores valores de massa de agregados na classe de diâmetro 2,00 mm, em comparação às áreas em SPD (Figura 1). Nas demais classes de agregados, a área de Cerradão apresentou valores iguais aos da área em SPD-ILP, tendo diferido apenas da área em SPD. Na profundidade de 5-10 cm, os maiores valores de massa de agregados foram encontrados na área de Cerradão, independentemente da classe de diâmetro avaliada. Entre as áreas cultivadas, os maiores valores de massa de agregados foram verificados para a área em SPD-ILP.

É provável que a maior massa de agregados na peneira de 2,00 $\mathrm{mm}$ tenha sido decorrente da ação de raízes (Six et al., 2004; Szakács, 2007) e hifas de fungos (Denef \& Six, 2005), tanto na área de Cerradão quanto nas áreas em SPD, o que beneficiou a formação de agregados de maior tamanho e maior estabilidade (Coutinho et al., 2010). Entre as áreas em SPD e SPD-ILP, os maiores valores de massa de agregados encontrados no SPD-ILP podem ter sido consequência do efeito agregante do sistema radicular da braquiária, que propiciou a formação de agregados mais estáveis em água, em comparação à área em SPD, sem braquiária (Salton et al., 2008; Kasper et al., 2009).

Tabela 1. Densidade do solo (Ds) em $\mathrm{Mg} \mathrm{m}^{-3}$, diâmetro médio ponderado (DMP) e diâmetro médio geométrico (DMG) de agregados em mm, e matéria orgânica leve (MOL) em $\mathrm{g} \mathrm{kg}^{-1}$, nas camadas 0-5 e 5-10 cm, nos diferentes sistemas de uso do $\operatorname{solo}^{(1)}$.

\begin{tabular}{|c|c|c|c|c|c|c|c|c|}
\hline \multirow[t]{2}{*}{ Sistema $^{(2)}$} & \multicolumn{4}{|c|}{$0-5 \mathrm{~cm}$} & \multicolumn{4}{|c|}{$5-10 \mathrm{~cm}$} \\
\hline & Ds & DMP & DMG & MOL & Ds & DMP & DMG & MOL \\
\hline$\overline{\mathrm{SPD}}$ & $0,94 a$ & $4,364 c$ & $3,383 c$ & $7,44 b$ & $1,06 \mathrm{a}$ & $3,406 \mathrm{c}$ & $2,134 \mathrm{c}$ & $1,98 b$ \\
\hline SPD-ILP & $0,89 \mathrm{~b}$ & $4,593 b$ & $3,832 b$ & $7,24 b$ & $0,97 \mathrm{~b}$ & $4,077 \mathrm{~b}$ & $2,752 b$ & $3,44 a$ \\
\hline Cerradão & $0,69 \mathrm{c}$ & 4,901a & $4,543 \mathrm{a}$ & $15,12 \mathrm{a}$ & $0,89 \mathrm{c}$ & $4,851 \mathrm{a}$ & $4,408 \mathrm{a}$ & $3,88 \mathrm{a}$ \\
\hline
\end{tabular}

${ }^{(1)}$ Médias, de quatro repetições, seguidas de letras iguais, nas colunas, não diferem pelo teste t, a 5\% de probabilidade. ${ }^{(2)}$ SPD, sistema plantio direto (girassolsoja-milho-algodão); SPD-ILP, sistema plantio direto com integração lavoura-pecuária (milho+braquiária-feijão-algodão-soja); Cerradão, condição original do solo. 
Na profundidade de 5-10 $\mathrm{cm}$, os maiores valores de massa de agregados na área em SPD-ILP (Figura 1), em comparação à área em SPD, podem estar relacionados indiretamente aos teores de MOL, ou seja, a maior quantidade de raízes (braquiária) que promovem a agregação reflete nos maiores valores de MOL, pois as raízes são matéria orgânica recente no solo. De acordo com Denef et al. (2007), a entrada de resíduos vegetais (MOL) estimula a reciclagem (manutenção) de agregados $>0,25 \mathrm{~mm}$, que são importantes para a estabilização da MOS ao longo do tempo. Portanto, a agregação do solo foi beneficiada no SPD-ILP pela maior quantidade de raízes da braquiária, o que culminou em maiores teores de MOL e acarretou

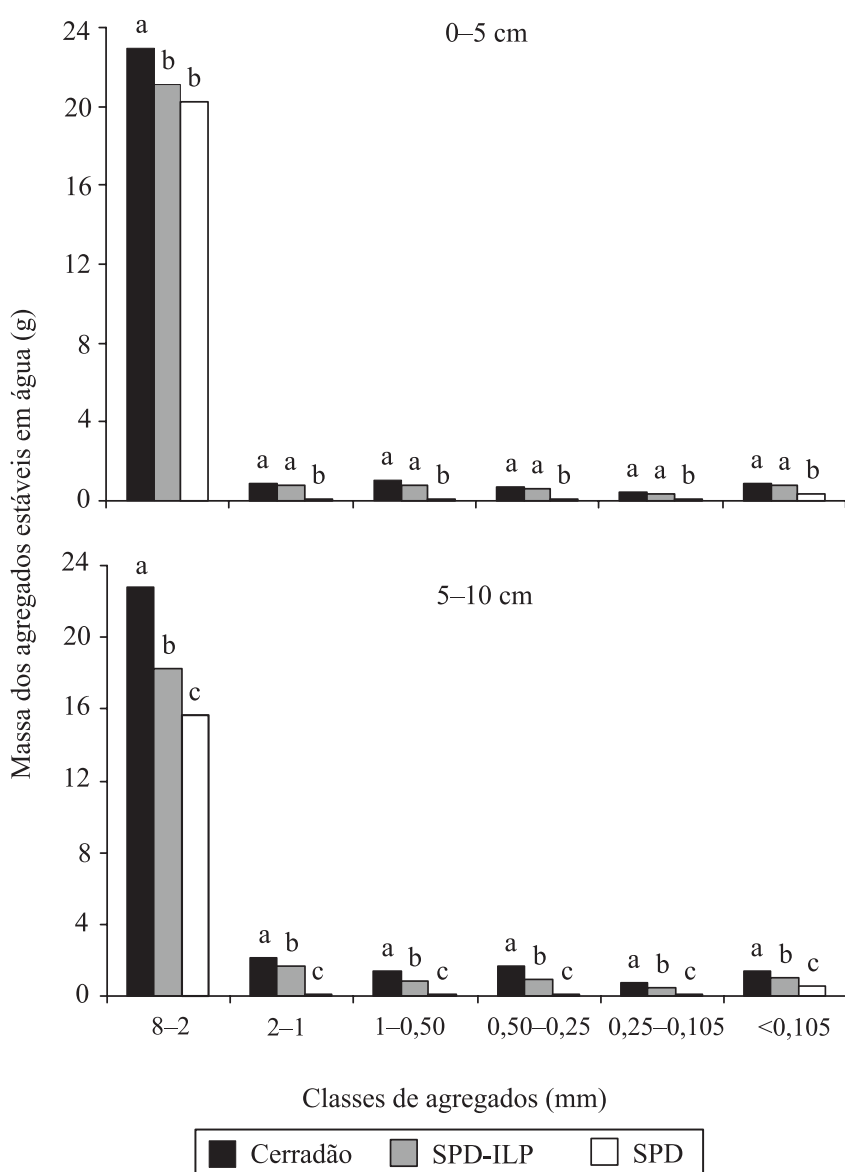

Figura 1. Distribuição dos agregados estáveis em água por classe de diâmetro, nas camadas 0-5 e 5-10 cm, nos diferentes sistemas de uso do solo. Médias, de quatro repetições, seguidas de letras iguais, na coluna, não diferem pelo teste $\mathrm{t}$, a $5 \%$ de probabilidade. SPD, sistema plantio direto (girassol-soja-milho-algodão); SPD-ILP, sistema plantio direto com integração lavoura-pecuária (milho+braquiáriafeijão-algodão-soja); Cerradão, condição original do solo. maiores índices de DMP e DMG (Tabela 1), em comparação ao SPD, sem braquiária.

A área em SPD-ILP, com maior estabilidade de agregados (DMPe DMG), promoveu a mitigação de $\mathrm{CO}_{2}$, ao conferir uma melhor proteção contra a decomposição do carbono orgânico (Szakács, 2007). A maior massa de agregados $>0,25 \mathrm{~mm}$ no SPD-ILP $(5-10 \mathrm{~cm})$ também sugere maior proteção do carbono orgânico contra a decomposição microbiana (Six et al., 2000).

Os maiores teores de $\mathrm{C}$ e $\mathrm{N}$ foram encontrados na área de Cerradão, independentemente da classe de agregado avaliada (Tabela 2). A intensa deposição de serapilheira, em virtude da condição natural do solo e da estabilidade do sistema, principalmente estabilidade física, com maiores valores de massa de agregados na classe de 8-2 mm (Figura 1), culminou em maiores valores de DMP e DMG (Tabela 1), que, por sua vez, acarretaram maior proteção dos teores de $\mathrm{C}$ e $\mathrm{N}$ no interior dos agregados.

Entre as áreas cultivadas, o SPD-ILP apresentou maiores teores de COT, com exceção da classe de 0,5-0,25 mm, que não apresentou diferença, e de $\mathrm{N}$, com exceção das classes de 8-2 e 0,5-0,25 mm, que não apresentaram diferenças em comparação à área em SPD, sem braquiária. Os maiores teores de COT

Tabela 2. Carbono orgânico total, nitrogênio, relação $\mathrm{C} / \mathrm{N}$ e abundância natural de ${ }^{15} \mathrm{~N}(\%$ ) , em agregados de Latossolo, em diferentes sistemas de uso do solo ${ }^{(1)}$.

\begin{tabular}{lccccc}
\hline Sistema $^{(2)}$ & \multicolumn{5}{c}{ Classes de agregados $(\mathrm{mm})$} \\
\cline { 2 - 6 } & $8-2$ & $2-1$ & $1-0,50$ & $0,50-0,25$ & $0,25-0,105$ \\
\hline CPD & $30,30 \mathrm{Ca}$ & $26,49 \mathrm{Ca}$ & $23,83 \mathrm{Ca}$ & $22,61 \mathrm{Ba}$ & $23,62 \mathrm{Ca}$ \\
SPD-ILP & $36,92 \mathrm{Ba}$ & $31,87 \mathrm{Bb}$ & $31,60 \mathrm{Bb}$ & $28,86 \mathrm{Bc}$ & $28,06 \mathrm{Bc}$ \\
Cerradão & $51,68 \mathrm{Aa}$ & $54,95 \mathrm{Aa}$ & $44,61 \mathrm{Ab}$ & $52,46 \mathrm{Aa}$ & $36,06 \mathrm{Ac}$ \\
\hline \multicolumn{5}{c}{ Nitrogênio $\left(\mathrm{g} \mathrm{kg}^{-1}\right)$} \\
SPD & $2,29 \mathrm{Ba}$ & $1,69 \mathrm{Ca}$ & $1,64 \mathrm{Ca}$ & $1,44 \mathrm{Ba}$ & $1,54 \mathrm{Ca}$ \\
SPD-ILP & $2,48 \mathrm{Ba}$ & $2,05 \mathrm{Bb}$ & $2,04 \mathrm{Bb}$ & $1,91 \mathrm{Bb}$ & $1,89 \mathrm{Bb}$ \\
Cerradão & $3,64 \mathrm{Aab}$ & $3,67 \mathrm{Aa}$ & $3,22 \mathrm{Abc}$ & $3,18 \mathrm{Ac}$ & $2,59 \mathrm{Ad}$ \\
\hline \multicolumn{5}{c}{ Relação C/N } \\
SPD & $14,81 \mathrm{Ab}$ & $15,73 \mathrm{Aa}$ & $15,86 \mathrm{Aa}$ & $15,81 \mathrm{Aa}$ & $15,43 \mathrm{Aa}$ \\
SPD-ILP & $14,79 \mathrm{Ac}$ & $15,48 \mathrm{Aa}$ & $15,44 \mathrm{Ba}$ & $15,10 \mathrm{Bb}$ & $14,82 \mathrm{Ac}$ \\
Cerradão & $14,12 \mathrm{Ba}$ & $14,75 \mathrm{Ba}$ & $14,61 \mathrm{Ca}$ & $14,35 \mathrm{Ca}$ & $13,98 \mathrm{Ba}$ \\
\hline \multicolumn{5}{c}{$\mathrm{Abundância} \mathrm{natural} \mathrm{de}$} & \\
\hline \multicolumn{5}{c}{$\mathrm{N}(\%)$} \\
SPD & $5,50 \mathrm{Aa}$ & $5,98 \mathrm{Aa}$ & $6,22 \mathrm{Aa}$ & $5,99 \mathrm{Aa}$ & $5,73 \mathrm{Aa}$ \\
SPD-ILP & $5,91 \mathrm{Aa}$ & $5,65 \mathrm{Aa}$ & $5,80 \mathrm{Aa}$ & $6,01 \mathrm{Aa}$ & $5,92 \mathrm{Aa}$ \\
Cerradão & $6,21 \mathrm{Aa}$ & $5,01 \mathrm{Aa}$ & $4,68 \mathrm{Ba}$ & $5,03 \mathrm{Aa}$ & $5,25 \mathrm{Aa}$ \\
\hline
\end{tabular}

(1)Médias seguidas de letras iguais, minúsculas na linha e maiúsculas na coluna, não diferem pelo teste $t$, a $5 \%$ de probabilidade. ${ }^{(2)} \mathrm{SPD}$, sistema plantio direto (girasol-soja-milho-algodão); SPD-ILP, sistema plantio direto com integração lavoura-pecuária (milho+braquiária-feijão-algodãosoja); Cerradão, condição original do solo. 
e N na área em SPD-ILP ocorreram, provavelmente, em virtude do uso da braquiária, que propicia a deposição de resíduos culturais de maior relação $\mathrm{C} / \mathrm{N}$, o que acarreta uma degradação mais lenta e favorece o acúmulo de COT e N nos agregados do solo. Portanto, a ILP foi mais eficiente em aumentar os teores de COT e $\mathrm{N}$ nos agregados do solo em comparação ao SPD, sem ILP.

Entre as classes de agregados, o SPD não apresentou significância $(\mathrm{p}<0,05)$ para os teores de COT e N. No SPD-ILP, observaram-se maiores teores de COT e $\mathrm{N}$ na classe de maior diâmetro (8-2 mm), e não foram verificadas diferenças no teor de $\mathrm{N}$ entre as demais classes de agregados. Nas classes de 2-1 e 1-0,50 mm, verificaram-se os maiores teores de COT em comparação às classes de $0,5-0,25$ e $0,25-0,105 \mathrm{~mm}$. A área em SPD-ILP apresentou padrão semelhante ao da área de Cerradão, ou seja, maiores teores de COT e $\mathrm{N}$ nos macroagregados $(>0,25 \mathrm{~mm})$ e menores nos micro $(<0,25 \mathrm{~mm})$ (Tabela 2$)$.

Os maiores teores de COT e $\mathrm{N}$ nos agregados e a melhoria da qualidade física do solo (menores valores de Ds, maiores valores de DMP e DMG, e agregados maiores que $8,00-2,00 \mathrm{~mm}$ ) encontrados na área em SPD-ILP demonstram que a ILP tem potencial para reduzir as emissões de gases de efeito estufa, propiciar maior estabilidade à produção das culturas anuais e melhorar o aproveitamento da água e dos nutrientes nas atividades produtivas (Franchini et al., 2010).

Quanto à relação $\mathrm{C} / \mathrm{N}$, foram observados menores valores na área de Cerradão, que podem ser explicados pelos maiores teores de $\mathrm{N}$ encontrados na área. Passos et al. (2007) obtiveram resultados diferentes, ao comparar os valores da relação $\mathrm{C} / \mathrm{N}$ em agregados em cultivo com milho, durante 30 anos, em áreas de Cerradão, em MG. Os autores verificaram maiores valores de $\mathrm{C} / \mathrm{N}$ na área com milho em decorrência da qualidade do material vegetal adicionado (milho, $\mathrm{C}_{4}$ ). No Cerradão, o sistema já está estável, com a relação $\mathrm{C}: \mathrm{N}$ tendendo a ser mais próxima da biomassa microbiana.

As relações $\mathrm{C} / \mathrm{N}$ das áreas cultivadas apresentaram valores semelhantes, com diferenças apenas nas classes de agregados de $1-0,5$ e $0,5-0,25 \mathrm{~mm}$, com maiores valores na área em SPD. Entre as classes de agregados, para a área de Cerradão, a relação $\mathrm{C} / \mathrm{N}$ não apresentou significância $(p<0,05)$, provavelmente em função da maior estabilidade deste sistema natural, o que indica que o COT e o $\mathrm{N}$ variam em proporções relativamente iguais nas diferentes classes de diâmetros de agregados, como pode ser observado na Tabela 2 .

De maneira geral, entre as classes de agregados nas áreas cultivadas, observaram-se os menores valores de $\mathrm{C} / \mathrm{N}$ na classe de $8-2 \mathrm{~mm}$, não tendo sido verificadas grandes variações nas demais classes. As menores relações $\mathrm{C} / \mathrm{N}$ na classe de maior diâmetro são decorrentes dos maiores teores de COT, resultado que corrobora os de Passos et al. (2007).

Para os teores de $\delta^{15} \mathrm{~N}$, verificou-se que apenas a classe de diâmetro $0,50 \mathrm{~mm}$ apresentou diferenças entre os sistemas de manejo, e os menores teores foram encontrados na área de Cerradão; não foram verificadas diferenças entre as áreas cultivadas. Os maiores teores de $\delta^{15} \mathrm{~N}$ encontrados nas áreas cultivadas podem ter sido consequência das transformações de $\mathrm{N}$-orgânico em N-mineral e também da adição de adubos nitrogenados. Assim, conforme ocorrem as reações de mineralização, nitrificação, denitrificação e volatilização, a MOS restante torna-se enriquecida em átomos de $\delta^{15} \mathrm{~N}$ (Bustamante et al., 2004).

Ao avaliar o teor de $\delta^{15} \mathrm{~N}$ de áreas de Cerradão e de Caatinga em solos da Chapada do Araripe, CE, Mendonça et al. (2010) observaram que os menores valores de $\delta^{15} \mathrm{~N}$ estavam associados à maior quantidade de MOS e foram encontrados no solo de Cerradão. $\mathrm{Na}$ Caatinga, onde a superfície do solo apresentou menor conteúdo de matéria orgânica, encontraramse os maiores valores de $\delta^{15} \mathrm{~N}$, o que é um indício de mineralização. Os resultados encontrados no presente trabalho são semelhantes aos de Mendonça et al. (2010), pois, com exceção da classe de agregados de 2,00 mm, observou-se que a área de Cerradão apresentou tendência de menores valores de $\delta^{15} \mathrm{~N}$ e maiores de COT, enquanto nas áreas em SPD, foi observado padrão contrário.

Os valores de $\delta^{13} \mathrm{C}(\%)$ da área de Cerradão (média de -26,79\%o) são indicação da predominância de plantas de ciclo fotossintético $\mathrm{C}_{3}$. Para a área em SPD, observou-se valor médio entre as classes de agregados de $-17,50 \%$, e, para a área em SPD-ILP, valor médio de $-19,10 \%$ (Tabela 3 ). Os valores de $\delta^{13} \mathrm{C}$ (\%) encontrados nas áreas cultivadas indicam que houve uma mudança da vegetação original (Cerradão, plantas $\mathrm{C}_{3}$, com valores que variam entre -24 a $-34 \%$ o) para vegetação de ciclo fotossintético $\mathrm{C}_{4}$, com valores entre -6 a $-19 \%$. 
Tabela 3. Abundância natural de $\delta^{13} \mathrm{C}(\%)$ em agregados de Latossolo, em diferentes sistemas de uso do solo em Montividiu, GO.

\begin{tabular}{lccccc}
\hline Sistema $^{(1)}$ & \multicolumn{5}{c}{ Classes de agregados $(\mathrm{mm})$} \\
\cline { 2 - 6 } & $8-2$ & $2-1$ & $1-0,5$ & $0,5-0,25$ & $0,25-0,105$ \\
\hline SPD & $-18,13$ & $-17,49$ & $-17,09$ & $-17,22$ & $-17,56$ \\
SPD-ILP & $-19,59$ & $-18,90$ & $-18,80$ & $-18,96$ & $-19,24$ \\
Cerradão & $-26,64$ & $-26,90$ & $-26,97$ & $-26,77$ & $-26,69$ \\
\hline
\end{tabular}

${ }^{(1)} \mathrm{SPD}$, sistema plantio direto (girasol-soja-milho-algodão); SPD-ILP, sistema plantio direto com integração lavoura-pecuária (milho+braquiáriafeijão-algodão-soja); Cerradão, condição original do solo.

Os valores de $\delta^{13} \mathrm{C}$ das áreas em SPD foram significativamente maiores que os verificados na área de Cerradão. Os valores de $\delta^{13} \mathrm{C}$ encontrados refletem a vegetação instalada, principalmente o uso de plantas de ciclo fotossintético $\mathrm{C}_{4}$, tais como o milho+braquiária na área em SPD-ILP, e somente o milho na área em SPD. Os maiores valores de DMP e massa de agregados nas duas profundidades avaliadas (com exceção da classe de 8-2 mm, para a profundidade de $0-5 \mathrm{~cm}$ ) na área de ILP são, provavelmente, decorrentes do uso das plantas $\mathrm{C}_{4}$, que contribuem para a formação e a estabilização dos agregados estáveis em água, com destaque para a área com braquiária.

\section{Conclusão}

O sistema plantio direto com integração lavoura-pecuária favorece o aumento dos índices de agregação do solo na camada $0-10 \mathrm{~cm}$, dos teores de matéria orgânica leve na camada $5-10 \mathrm{~cm}$, da massa de agregados estáveis em água na camada $5-10 \mathrm{~cm}$, dos teores de carbono orgânico total e de $\mathrm{N}$ dos agregados na camada $0-5 \mathrm{~cm}$, em comparação ao sistema de plantio direto, sem braquiária.

\section{Agradecimentos}

À Fundação Agricultura Sustentável, pelo apoio financeiro; ao Conselho Nacional de Desenvolvimento Científico e Tecnológico e à Fundação de Amparo à Pesquisa do Estado do Rio de Janeiro, pela concessão de bolsa de Doutorado ao primeiro autor; à Coordenação do Curso de Pós-Graduação em Agronomia, Ciência do Solo, da Universidade Federal Rural do Rio de Janeiro, pelo auxílio prestado; e ao Instituto Federal de Educação, Ciência e Tecnologia Goiano, pelo auxílio na coleta das amostras de solo.

\section{Referências}

ANDERSON, J.M.; INGRAM, J.S.I. (Ed.). Tropical soil biology and fertility: a handbook of methods. Wallingford: $C A B$ International, 1989. 171p.

BARTLETT, M.S. The square root-transformation in the analysis of variance. Journal of the Royal Statistical Society, v.3, p.68-78, 1936.

BUSTAMANTE, M.M.C.; MARTINELLI, L.A.; SILVA, D.A.; CAMARGO, P.B.; KLINK, C.A.; DOMINGUES, T.F.; SANTOS, R.V. ${ }^{15} \mathrm{~N}$ natural abundance in woody plants and soils of central Brazil savannas (cerrado). Ecological Applications, v.14, p.200-213, 2004.

CLAESSEN, M.E.C. (Org.). Manual de métodos de análise de solo. 2.ed. rev. atual. Rio de Janeiro: Embrapa-CNPS, 1997. 212p. (Embrapa-CNPS. Documentos, 1).

COUTINHO, F.S.; LOSS, A.; PEREIRA, M.G.; RODRIGUES JUNIOR, D.J.; TORRES, J.L.R. Estabilidade de agregados e distribuição do carbono em Latossolo sob sistema plantio direto, Uberaba, Minas Gerais. Comunicata Scientiae, v.1, p.100-105, 2010.

DENEF, K.; SIX, J. Clay mineralogy determines the importance of biological versus abiotic processes for macroaggregate formation and stabilization. European Journal of Soil Science, v.56, p.469-479, 2005.

DENEF, K.; ZOTARELLI, L.; BODDEY, R.M.; SIX, J. Microaggregate-associated carbon as a diagnostic fraction for management-induced changes in soil organic carbon in two Oxisols. Soil Biology and Biochemistry, v.39, p.1165-1172, 2007.

ESPECIAL Embrapa: integração lavoura, pecuária e floresta. 2009. Disponível em: <www.cnpc.org.br/arquivos/integlavpecflo.pdf>. Acesso em: 22 jun. 2010.

FONSECA, G.C.; CARNEIRO, M.A.C.; COSTA, A.R. da; OLIVEIRA, G.C. de; BALBINO, L.C. Atributos físicos, químicos e biológicos de Latossolo Vermelho distrófico de Cerrado sob duas rotações de cultura. Pesquisa Agropecuária Tropical, v.37, p.22-30, 2007.

FRANCHINI, J.C.; DEBIASI, H.; WRUCK, F.J.; SKORUPA, L.A.; WINK, N.N.; GUISOLPHI, I.J.; CAUMO, A.L.; HATORI, T. Integração lavoura-pecuária: alternativa para diversificação e redução do impacto ambiental do sistema produtivo no Vale do Rio Xingu. Londrina: Embrapa Soja, 2010. 20p. (Embrapa Soja. Circular técnica, 77).

GONÇALVES, S.L.; FRANCHINI, J.C. Integração lavoura-pecuária. Londrina: Embrapa Soja, 2007. 7p. (Embrapa Soja. Circular técnica, 44).

KASPER, M.; BUCHAN, G.D.; MENTLER, A.; BLUM, W.E.H. Influence of soil tillage systems on aggregate stability and the distribution of $\mathrm{C}$ and $\mathrm{N}$ in different aggregate fractions. Soil and Tillage Research, v.105, p.192-199, 2009.

MACEDO, M.C.M. Integração lavoura e pecuária: o estado da arte e inovações tecnológicas. Revista Brasileira de Zootecnia, v.38, p.133-146, 2009.

MENDONÇA, L.A.R.; FRISCHKORN, H.; SANTIAGO, M.F; CAMARGO, P.B. de; LIMA, J.O.G. de; MENDES FILHO, J. 
Identificação de mudanças florestais por ${ }^{13} \mathrm{C}$ e ${ }^{15} \mathrm{~N}$ dos solos da Chapada do Araripe/Ceará. Revista Brasileira de Engenharia Agrícola e Ambiental, v.14, p.314-319, 2010.

MORETI, D.; ALVES, M.C.; VALÉRIO FILHO, W.V.; CARVALHO, M. de P. e. Atributos químicos de um Latossolo Vermelho sob diferentes sistemas de preparo, adubações e plantas de cobertura. Revista Brasileira de Ciência do Solo, v.31, p.167-175, 2007.

PASSOS, R.R.; RUIZ, H.A.; CANTARUTTI, R.B.; MENDONÇA, E. de S. Carbono orgânico e nitrogênio em agregados de um Latossolo Vermelho sob duas coberturas vegetais. Revista Brasileira de Ciência do Solo, v.31, p.1109-1118, 2007.

PEREIRA, M.G.; LOSS, A.; BEUTLER, S.J.; TORRES, J.L.R. Carbono, matéria orgânica leve e fósforo remanescente em diferentes sistemas de manejo de solo. Pesquisa Agropecuária Brasileira, v.45, p.508-514, 2010.

SALTON, J.C.; MIELNICZUK, J.; BAYER, C.; BOENI, M.; CONCEIÇÃO, P.C.; FABRICIO, A.C.; MACEDO, M.C.M.; BROCH, D.L. Agregação e estabilidade de agregados do solo em sistemas agropecuários em Mato Grosso do Sul. Revista Brasileira de Ciência do Solo, v.32, p.11-21, 2008.
SANTOS, H.G. dos; JACOMINE, P.K.T.; ANJOS, L.H.C. dos; OLIVEIRA, V.A. de; OLIVEIRA, J.B. de; COELHO, M.R.; LUMBRERAS, J.F.; CUNHA, T.J.F. (Ed.). Sistema brasileiro de classificação de solos. 2.ed. Rio de Janeiro: Embrapa Solos, 2006. $306 \mathrm{p}$.

SIX, J.; BOSSUYT, H.; DEGRYZE, S.; DENEF, K. A history of research on the link between (micro) aggregates, soil biota, and soil organic matter dynamics. Soil and Tillage Research, v.79, p.7-31, 2004.

SIX, J.; ELLIOTT, E.T.; PAUSTIAN, K. Soil macroaggregate turnover and microaggregate formation: a mechanism for $\mathrm{C}$ sequestration under no-tillage agriculture. Soil Biology and Biochemistry, v.32, p.2099-2103, 2000.

SZAKÁCS, G.G.J. Estoques de carbono e agregados do solo cultivado com cana-de-açúcar: efeito da palhada e do clima no centro-sul do Brasil. 2007. 105p. Tese (Doutorado) - Centro de Energia Nuclear na Agricultura, Piracicaba.

YOODER, R.E. A direct method of aggregate analysis of soil and a study of the physical nature of erosion losses. Journal of the American Society of Agronomy, v.28, p.337-351, 1936.

Recebido em 24 de junho de 2010 e aprovado em 18 de agosto de 2011 